\title{
Algebraic cycles on Jacobian varieties
}

\author{
Arnaud Beauville
}

\begin{abstract}
Let $J$ be the Jacobian of a smooth curve $C$ of genus $g$, and let $A(J)$ be the ring of algebraic cycles modulo algebraic equivalence on $J$, tensored with $\mathbb{Q}$. We study in this paper the smallest $\mathbb{Q}$-vector subspace $R$ of $A(J)$ which contains $C$ and is stable under the natural operations of $A(J)$ : intersection and Pontryagin products, pull back and push down under multiplication by integers. We prove that this 'tautological subring' is generated (over $\mathbb{Q}$ ) by the classes of the subvarieties $W_{1}=C, W_{2}=C+C, \ldots, W_{g-1}$. If $C$ admits a morphism of degree $d$ onto $\mathbb{P}^{1}$, we prove that the last $d-1$ classes suffice.
\end{abstract}

\section{Introduction}

Let $C$ be a compact Riemann surface of genus $g$. Its Jacobian variety $J$ carries a number of natural subvarieties, defined up to translation: first of all the curve $C$ embeds into $J$, then we can use the group law of $J$ to form $W_{2}=C+C, W_{3}=C+C+C, \ldots$ until $W_{g-1}$ which is a theta divisor on $J$. Then we can intersect these subvarieties, add again, pull back or push down under multiplication by integers, and so on. Thus we get a rather large number of algebraic subvarieties which live naturally in $J$.

If we look at the classes obtained in this way in rational cohomology, the result is disappointing. We just find the subalgebra of $H^{*}(J, \mathbb{Q})$ generated by the class $\theta$ of the theta divisor. In fact, the polynomials in $\theta$ are the only algebraic cohomology classes which live on a generic Jacobian. The situation becomes more interesting if we look at the $\mathbb{Q}$-algebra $A(J)$ of algebraic cycles modulo algebraic equivalence on $J$; here a result of Ceresa [Cer83] implies that, for a generic curve $C$, the class of $W_{g-p}$ in $A^{p}(J)$ is not proportional to $\theta^{p}$ for $2 \leqslant p \leqslant g-1$. This leads naturally to investigate the 'tautological subring' of $A(J)$, that is, the smallest $\mathbb{Q}$-vector subspace $R$ of $A(J)$ which contains $C$ and is stable under the natural operations of $A(J)$ : intersection and Pontryagin products (see start of $\S 2$ ), pull back and push down under multiplication by integers. Our main result states that this space is not too complicated. Let $w^{p} \in A^{p}(J)$ be the class of $W_{g-p}$. Then we can state the following theorem.

THEOREM.

a) $R$ is the sub- $\mathbb{Q}$-algebra of $A(J)$ generated by $w^{1}, \ldots, w^{g-1}$.

b) If $C$ admits a morphism of degree $d$ onto $\mathbb{P}^{1}, R$ is generated by $w^{1}, \ldots, w^{d-1}$.

In particular we see that $R$ is finite-dimensional, a fact which does not seem to be a priori obvious (the space $A(J)$ is known to be infinite-dimensional for $C$ generic of genus 3, see [Nor89]).

The proof rests in an essential way on the properties of the Fourier transform, a $\mathbb{Q}$-linear automorphism of $A(J)$ with remarkable properties. We recall these properties in $\S 1$; in $\S 2$ we look at the case of Jacobian varieties, computing in particular the Fourier transform of the class of $C$

Received 22 April 2002, accepted in final form 3 June 2002.

2000 Mathematics Subject Classification 14C15, 14C25, 14K12.

Keywords: algebraic cycles, algebraic equivalence, Jacobian.

This journal is (C) Foundation Compositio Mathematica 2004. 


\section{A. Beauville}

in $A(J)$. This is the main ingredient in the proof of part a of the Theorem, which we give in $\S 3$. Part $b$ turns out to be an easy consequence of a result of Colombo and van Geemen [CG93]; this is explained in $\S 4$, together with a few examples.

\section{Algebraic cycles on abelian varieties}

2.1 Let $X$ be an abelian variety over $\mathbf{C}$. We will denote by $p$ and $q$ the two projections of $X \times X$ onto $X$, and by $m: X \times X \rightarrow X$ the addition map.

Let $A(X)$ be the group of algebraic cycles on $X$ modulo algebraic equivalence, tensored with $\mathbb{Q}$. It is a $\mathbb{Q}$-vector space, graded by the codimension of the cycle classes. It carries two natural multiplication laws $A(J) \otimes_{\mathbb{Q}} A(J) \rightarrow A(J)$, which are associative and commutative: the intersection product, which is homogeneous with respect to the graduation, and the Pontryagin product, defined by

$$
x * y:=m_{*}\left(p^{*} x \cdot q^{*} y\right),
$$

which is homogeneous of degree $-g$. If $Y$ and $Z$ are subvarieties of $X$, the cycle class $[Y] *[Z]$ is equal to $(\operatorname{deg} \mu)[Y+Z]$ if the addition map $\mu: Y \times Z \rightarrow Y+Z$ is generically finite, and is zero otherwise.

2.2 For $k \in \mathbb{Z}$, we will still denote by $k$ the endomorphism $x \mapsto k x$ of $X$. According to [Bea86], there is a second graduation on $A(X)$, leading to a bigraduation

$$
A(X)=\bigoplus_{s, p} A^{p}(X)_{(s)}
$$

such that

$$
k^{*} x=k^{2 p-s} x, \quad k_{*} x=k^{2 g-2 p+s} x \quad \text { for } x \in A^{p}(X)_{(s)} .
$$

Both products are homogeneous with respect to the second graduation. We have $A^{p}(X)_{(s)}=0$ for $s<p-g$ or $s \geqslant p$ (use [Bea86, Proposition 4]). It is conjectured that negative degrees actually do not occur; this will not concern us here, as we will only consider cycles in $A(X)_{(s)}$ for $s \geqslant 0$.

2.3 A crucial tool in what follows will be the Fourier transform for algebraic cycles, defined in [Bea83]. Let us recall briefly the results we will need, the proofs can be found in [Bea83] and [Bea86]. We will concentrate on the case of a principally polarized abelian variety $(X, \theta)$, and use the polarization to identify $X$ with its dual abelian variety.

Let $\ell:=p^{*} \theta+q^{*} \theta-m^{*} \theta \in A^{1}(X \times X)$; this is the class of the Poincaré line bundle $\mathcal{L}$ on $X \times X$. The Fourier transform $\mathcal{F}: A(X) \rightarrow A(X)$ is defined by $\mathcal{F} x=q_{*}\left(p^{*} x \cdot e^{\ell}\right)$. It satisfies the following properties:

i) $\mathcal{F} \circ \mathcal{F}=(-1)^{g}(-1)^{*}$;

ii) $\mathcal{F}(x * y)=\mathcal{F} x \cdot \mathcal{F} y$ and $\mathcal{F}(x \cdot y)=(-1)^{g} \mathcal{F} x * \mathcal{F} y$;

iii) $\mathcal{F} A^{p}(X)_{(s)}=A^{g-p+s}(X)_{(s)}$;

iv) let $x \in A(X)$; put $\bar{x}=(-1)^{*} x$. Then $\mathcal{F} x=e^{\theta}\left(\left(\bar{x} e^{\theta}\right) * e^{-\theta}\right)$.

Let us prove property iv, which is not explicitly stated in [Bea83] or [Bea86]. Replacing $\ell$ by its definition, we get $\mathcal{F} x=e^{\theta} q_{*}\left(p^{*}\left(x e^{\theta}\right) \cdot e^{-m^{*} \theta}\right)$. Let $\omega$ be the automorphism of $A \times A$ defined by $\omega(a, b)=(-a, a+b)$. We have $p \circ \omega=-p, q \circ \omega=m$, and $m \circ \omega=q$. Hence

$$
\mathcal{F} x=e^{\theta} q_{*} \omega_{*} \omega^{*}\left(p^{*}\left(x e^{\theta}\right) \cdot e^{-m^{*} \theta}\right)=e^{\theta} m_{*}\left(p^{*}\left(\bar{x} e^{\theta}\right) \cdot q^{*} e^{-\theta}\right)=e^{\theta}\left(\left(\bar{x} e^{\theta}\right) * e^{-\theta}\right),
$$

and thus property iv is proved. 


\section{Algebraic CyCles on JaCobian VARIETIES}

\section{The Fourier transform on a Jacobian}

3.1 From now on we take for our abelian variety the Jacobian $(J, \theta)$ of a smooth projective curve $C$ of genus $g$. We choose a base point $o \in C$, which allows us to define an embedding $\varphi: C \longleftrightarrow J$ by $\varphi(p)=\mathcal{O}_{C}(p-o)$. Since we are working modulo algebraic equivalence, all our constructions will be independent of the choice of the base point.

We will denote simply by $C$ the class of $\varphi(C)$ in $A^{g-1}(J)$. For $0 \leqslant d \leqslant g$, we put $w^{g-d}:=$ $(1 / d !) C^{* d} \in A^{g-d}(J)$; it is the class of the subvariety $W_{d}$ of $J$ parameterizing line bundles of the form $\mathcal{O}_{C}\left(E_{d}-d o\right)$, where $E_{d}$ is an effective divisor of degree $d$. We have $w^{1}=\theta$ by the Riemann theorem, $w^{g-1}=C$, and $w^{g}$ is the class of a point. We define the Newton polynomials in the classes $w^{i}$ by

$$
N^{k}(w)=\frac{1}{k !} \sum_{i=1}^{g} \lambda_{i}^{k}
$$

in the ring obtained by adjoining to $A(J)$ the roots $\lambda_{1}, \ldots, \lambda_{g}$ of the equation $\lambda^{g}-\lambda^{g-1} w^{1}+\cdots+$ $(-1)^{g} w^{g}=0$. We have $N^{k}(w) \in A^{k}(J)$; for instance

$$
N^{1}(w)=\theta, \quad N^{2}(w)=\frac{1}{2} \theta^{2}-w^{2}, \quad N^{3}(w)=\frac{1}{6} \theta^{3}-\frac{1}{2} \theta \cdot w^{2}-\frac{1}{2} w^{3}, \ldots
$$

3.2 The class $N^{k}(w)$ is a polynomial in $w^{1}, \ldots, w^{k}$; conversely, $w^{k}$ is a polynomial in $N^{1}(w), \ldots$, $N^{k}(w)$.

Proposition 3.3. We have $-\mathcal{F} C=N^{1}(w)+N^{2}(w)+\cdots+N^{g-1}(w)$.

Proof. We use the notation of $\S 2$, and denote moreover by $\bar{p}, \bar{q}$ the projections of $C \times J$ onto $C$ and $J$. Consider the cartesian diagram

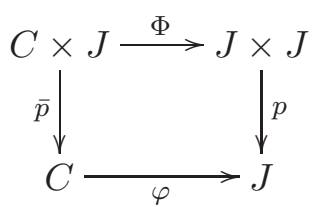

with $\Phi=\left(\varphi, 1_{J}\right)$. Put $\bar{\ell}:=\Phi^{*} \ell$. We have $p^{*} C \cdot e^{\ell}=\Phi_{*} 1 \cdot e^{\ell}=\Phi_{*} e^{\bar{\ell}}$, and therefore

$$
\mathcal{F} C=\bar{q}_{*} e^{\bar{\ell}}
$$

The line bundle $\overline{\mathcal{L}}:=\Phi^{*} \mathcal{L}$ is the Poincaré line bundle on $C \times J$ : that is, we have $\overline{\mathcal{L}}_{C \times\{\alpha\}}=\alpha$ for all $\alpha \in J$, and $\overline{\mathcal{L}}_{\{o\} \times J}=\mathcal{O}_{J}$. We will now work exclusively on $C \times J$, and suppress the bar above the letters $p, q, \mathcal{L}$ and $\ell$. We apply the Grothendieck-Riemann-Roch theorem to $q$ and $\mathcal{L}$. Since we are working modulo algebraic equivalence, the Todd class of $C$ is simply $1+(1-g)$. Let $i_{o}: J \longleftrightarrow C \times J$ be the map $\alpha \mapsto(o, \alpha)$; we have

$$
q_{*}\left(p^{*} o \cdot e^{\ell}\right)=q_{*} i_{o *} i_{o}^{*} e^{\ell}=i_{o}^{*} e^{\ell}=1,
$$

since $i_{o}^{*} \mathcal{L}$ is trivial. Thus

$$
\operatorname{ch} q ! \mathcal{L}=q_{*}\left(p^{*} \operatorname{Todd}(C) \cdot \operatorname{ch} \mathcal{L}\right)=q_{*} e^{\ell}-(g-1) .
$$

The Chern classes of $q ! \mathcal{L}$ are computed in [Mat61]: we have

$$
c(-q ! \mathcal{L})=1+w^{1}+\cdots+w^{g} .
$$

Putting things together we obtain

$$
\mathcal{F} C=q_{*} e^{\ell}=g-1-\operatorname{ch}\left(-q_{!} \mathcal{L}\right)=-\left(N^{1}(w)+N^{2}(w)+\cdots+N^{g}(w)\right) .
$$




\section{A. Beauville}

Let $C=\sum_{s=0}^{g-1} C_{(s)}$ be the decomposition of $C$ in $\bigoplus_{s} A^{g-1}(J)_{(s)}$. From Proposition 3.3, and properties iii and i in $\S 2$, we obtain a corollary.

Corollary 3.4. We have $N^{k}(w)=-\mathcal{F} C_{(k-1)} \in A^{k}(J)_{(k-1)}$ and $\mathcal{F}\left(N^{k}(w)\right)=(-1)^{g+k} C_{(k-1)}$.

Corollary 3.5. The $\mathbb{Q}$-subalgebra $R$ of $A(J)$ generated by $w^{1}, \ldots, w^{g-1}$ is bigraded. In particular, it is stable under the operations $k^{*}$ and $k_{*}$ for each $k \in \mathbb{Z}$.

Indeed $R$ is also generated by the elements $N^{1}(w), \ldots, N^{g-1}(w)$ given above, which are homogeneous for both graduations.

\section{Proof of the main result}

In order to prove part a of the Theorem, it remains to prove that the $\mathbb{Q}$-subalgebra $R$ of $A(J)$ generated by $w^{1}, \ldots, w^{g-1}$ is stable under the Pontryagin product. In view of property ii in $\S 2$, it suffices to prove the following.

Proposition 4.1. $R$ is stable under $\mathcal{F}$.

Proof. Let $\mathcal{F} R$ denote the image of $R$ under the Fourier transform; it is a vector space over $\mathbb{Q}$, stable under the Pontryagin product (property ii). We will prove that $\mathcal{F} R$ is stable under $\mathcal{F}$, that is, $\mathcal{F} \mathcal{F} R \subset \mathcal{F} R$; since $\mathcal{F} \mathcal{F} R=R$ (property i), this implies $R \subset \mathcal{F} R$, then $\mathcal{F} R \subset R$ by applying $\mathcal{F}$ again.

We observe that it is enough to prove that $\mathcal{F} R$ is stable under multiplication by $\theta$. Indeed, it is then stable under multiplication by $e^{\theta}$, and finally under $\mathcal{F}$ in view of property iv, $\mathcal{F} x=$ $e^{\theta}\left(\left(\bar{x} e^{\theta}\right) * e^{-\theta}\right)$.

Since the $\mathbb{Q}$-algebra $R$ is generated by the classes $N^{p}(w), \mathcal{F} R$ is spanned as a $\mathbb{Q}$-vector space by the elements

$$
\mathcal{F}\left(N^{p_{1}}(w) \cdots N^{p_{r}}(w)\right)= \pm C_{\left(p_{1}-1\right)} * \cdots * C_{\left(p_{r}-1\right)}
$$

(we are using property ii and Corollary 3.4).

Lemma 4.2. $\mathcal{F} R$ is spanned by the classes $\left(k_{1 *} C\right) * \cdots *\left(k_{r *} C\right)$, for all sequences $\left(k_{1}, \ldots, k_{r}\right)$ of positive integers.

Proof. For $k \in \mathbb{Z}$ we have from $\S 2$ that

$$
k_{*} C=\sum_{s=0}^{g-1} k^{2+s} C_{(s)} .
$$

Therefore

$$
\left(k_{1 *} C\right) * \cdots *\left(k_{r *} C\right)=\left(k_{1} \cdots k_{r}\right)^{2} \sum_{s_{1}, \ldots, s_{r}} k_{1}^{s_{1}} \cdots k_{r}^{s_{r}} C_{\left(s_{1}\right)} * \cdots * C_{\left(s_{r}\right)},
$$

where $\mathbf{s}=\left(s_{1}, \ldots, s_{r}\right)$ runs in $[0, g-1]^{r}$; this shows in particular that $\left(k_{1 *} C\right) * \cdots *\left(k_{r *} C\right)$ belongs to $\mathcal{F} R$. We claim that we can choose $g^{r} r$-tuples $\mathbf{k}=\left(k_{1}, \ldots, k_{r}\right)$ so that the matrix $\left(a_{\mathbf{k}, \mathbf{s}}\right)$ with entries $a_{\mathbf{k}, \mathbf{s}}=\left(k_{1}^{s_{1}} \cdots k_{r}^{s_{r}}\right)$ is invertible: if we take for instance the sequence of $r$-tuples $\mathbf{k}_{\ell}=\left(\ell, \ell^{g}, \ldots, \ell^{g^{r-1}}\right)$, for $1 \leqslant \ell \leqslant g^{r}$, we get for $\operatorname{det}\left(a_{\mathbf{k}, \mathbf{s}}\right)$ a non-zero Vandermonde determinant. Thus each element $C_{\left(s_{1}\right)} * \cdots * C_{\left(s_{r}\right)}$ is a $\mathbb{Q}$-linear combination of classes of the form $\left(k_{1 *} C\right) * \cdots *\left(k_{r *} C\right)$, which proves Lemma 4.2 .

We now return to the proof of Proposition 4.1. 


\section{Algebraic CyCles on Jacobian varieties}

Thus it suffices to prove that each product $\theta \cdot\left(\left(k_{1 *} C\right) * \cdots *\left(k_{r *} C\right)\right)$ belongs to $\mathcal{F} R$. We observe that $\left(k_{1 *} C\right) * \cdots *\left(k_{r *} C\right)$ is a multiple of the image of the composite map

$$
u: C^{r} \stackrel{\varphi}{\longrightarrow} J^{r} \stackrel{\mathbf{k}}{\longrightarrow} J^{r} \stackrel{m}{\longrightarrow} J
$$

where $\mathbf{k}=\left(k_{1}, \ldots, k_{r}\right), \boldsymbol{\varphi}=(\varphi, \ldots, \varphi)$ and $m$ is the addition morphism. Thus the class $\theta \cdot\left(\left(k_{1 *} C\right) *\right.$ $\left.\cdots *\left(k_{r *} C\right)\right)$ is proportional to $u_{*} u^{*} \theta$.

Let $p_{i}: J^{r} \rightarrow J$ (respectively $p_{i j}: J^{r} \rightarrow J^{2}$ ) denote the projection onto the $i$ th factor (respectively the $i$ th and $j$ th factors). In $A^{1}\left(J^{r}\right)$, we have

$$
m^{*} \theta=\sum_{i} p_{i}^{*} \theta-\sum_{i<j} p_{i j}^{*} \ell
$$

indeed for $r=2$ this is the definition of $\ell$, and the general case follows from the theorem of the cube. We have also $k_{i}^{*} \theta=k_{i}^{2} \theta$ and $\left(k_{i}, k_{j}\right)^{*} \ell=k_{i} k_{j} \ell$. Thus

$$
\mathbf{k}^{*} m^{*} \theta=\sum_{i} k_{i}^{2} p_{i}^{*} \theta-\sum_{i<j} k_{i} k_{j} p_{i j}^{*} \ell ;
$$

denoting by $q_{i}, q_{i j}$ the projections of $C^{r}$ onto $C$ and $C^{2}$, we find

$$
u^{*} \theta=\sum_{i} k_{i}^{2} q_{i}^{*} \varphi^{*} \theta-\sum_{i<j} k_{i} k_{j} q_{i j}^{*}(\varphi, \varphi)^{*} \ell
$$

Let $\Delta$ be the diagonal in $C^{2}$. The theorem of the square gives

$$
(\varphi, \varphi)^{*} \mathcal{L}=\mathcal{O}_{C^{2}}(\Delta-C \times o-o \times C) .
$$

Therefore $u^{*} \theta$ is algebraically equivalent to a linear combination of divisors of the form $q_{i}^{*} o$ and $q_{i j}^{*} \Delta$. Under $u_{*}$ each of these divisors is mapped to a multiple of the cycle $\left(l_{1 *} C\right) * \cdots *\left(l_{r-1 *} C\right)$, where the sequence $\left(l_{1} \cdots l_{r-1}\right)$ is $\left(k_{1}, \ldots, \widehat{k_{i}}, \ldots, k_{r}\right)$ in the first case and $\left(k_{1}, \ldots, \widehat{k_{i}}, \ldots, \widehat{k_{j}}, \ldots, k_{r}, k_{i}+k_{j}\right)$ in the second one (as usual the symbol $\widehat{k_{i}}$ means that $k_{i}$ is omitted). This proves our claim, and therefore Proposition 4.1 .

\section{5. d-gonal curves}

Proposition 5.1. Assume that the curve $C$ is $d$-gonal, that is, admits a degree $d$ morphism onto $\mathbb{P}^{1}$. We have $N^{k}(w)=0$ for $k \geqslant d$, and the $\mathbb{Q}$-algebra $R$ is generated by $w^{1}, \ldots, w^{d-1}$.

Proof. By now this is an immediate consequence of a result of Colombo and van Geemen, which says that for a $d$-gonal curve $C_{(s)}=0$ for $s \geqslant d-1$ [CG93, Proposition 3.6]. (Our class $C_{(s)}$ is denoted $\pi_{2 g-2-s} C$ in [CG93].) This implies $N^{k}(w)=0$ for $k \geqslant d$ [CG93, Proposition 3.2], so that $R$ is a polynomial ring in $N^{1}(w), \ldots, N^{d-1}(w)$, hence in $w^{1}, \ldots, w^{d-1}$ (from $\S 3$ ).

The case $d=2$ of Proposition 5.1 had already been observed by Collino [Col75].

Corollary 5.2. If $C$ is hyperelliptic, $R=\mathbb{Q}[\theta] /\left(\theta^{g+1}\right)$.

Corollary 5.3. If $C$ is trigonal, $R$ is generated by $\theta$ and the class $\eta=N^{2}(w)$ in $A^{2}(J)$. There exists an integer $k \leqslant g / 3$ such that

$$
R=\mathbb{Q}[\theta, \eta] /\left(\theta^{g+1}, \theta^{g-2} \eta, \ldots, \theta^{g+1-3 k} \eta^{k}, \eta^{k+1}\right) .
$$

Proof. By Proposition $5.1 R$ is generated by $\theta$ and $\eta$. For $p, s \in \mathbb{N}$, the class $\theta^{p-2 s} \eta^{s}$ is the only monomial in $\theta, \eta$ which belongs to $A^{p}(J)_{(s)}$; therefore it spans the $\mathbb{Q}$-vector space $R_{(s)}^{p}$ (in particular, this space is zero for $p<2 s$ ). This implies that the relations between $\theta$ and $\eta$ are monomial, that is, of the form $\theta^{r} \eta^{s}=0$ for some pairs $(r, s) \in \mathbb{N}^{2}$. 


\section{Algebraic CYCles ON JACOBIAN VARIETIES}

Similarly, as a $\mathbb{Q}$-algebra for the Pontryagin product, $R$ is generated by $C_{(0)}$ and $C_{(1)}$. The $\mathbb{Q}$-vector space $R_{(s)}^{p}$ is spanned by $C_{(0)}^{*(g-p-s)} * C_{(1)}^{* s}$, hence is zero for $p+s>g$. In particular we see that $\theta^{r} \eta^{s}=0$ as soon as $r+3 s>g$.

Let $k$ be the smallest integer such that $\eta^{k} \neq 0, \eta^{k+1}=0$. By what we have just seen the first relation implies $3 k \leqslant g$. Suppose we have $\theta^{r} \eta^{s}=0$ for some integers $r, s$ with $r+3 s \leqslant g$ and $s \leqslant k$. Then we have $R_{(s)}^{r+2 s}=0$ and $C_{(0)}^{*(g-r-3 s)} * C_{(1)}^{* s}=0$. Taking $*$-product with $C_{(0)}^{* r}$ we arrive at $C_{(0)}^{*(g-3 s)} * C_{(1)}^{* s}=0$, which implies $\eta^{s}=0$, contradicting the definition of $k$.

In the general case, since any curve of genus $g$ has a $g_{d}^{1}$ with $d \leqslant(g+3) / 2$ [ACGH85, ch. V, Theorem 1.1] we get a corollary.

Corollary 5.4. Put $d:=[(g+1) / 2]$. The $\mathbb{Q}$-algebra $R$ is generated by $w^{1}, \ldots, w^{d}$.

5.5 We may now ask how many of the classes $w^{i}$ are really needed to generate $R$. Since $N^{k}(w)$ belongs to $A^{k}(J)_{k-1}$, it is readily seen that it cannot be a polynomial in $N^{1}(w), \ldots, N^{k-1}(w)$ unless it is zero. Thus the question is to determine when these classes vanish. I know only two results in that direction: Ceresa's result [Cer83] implies that $N^{2}(w)$ is non-zero for a generic curve of genus $\geqslant 3$, and Fakhruddin proved that $N^{3}(w)$ is non-zero for a generic curve of genus $\geqslant 11$ [Fak96, Corollary 4.6]. It would be interesting to extend this to higher-codimensional classes.

\section{REFERENCES}

ACGH85 E. Arbarello, M. Cornalba, P. Griffiths and J. Harris, Geometry of algebraic curves I, Grundlehren der Mathematischen Wissenschaften, vol. 267 (Springer, Berlin, 1985).

Bea83 A. Beauville, Quelques remarques sur la transformation de Fourier dans l'anneau de Chow d'une variété abélienne, in Algebraic Geometry (Tokyo/Kyoto 1982), Lecture Notes in Mathematics, vol. 1016 (Springer, Berlin, 1983), 238-260.

Bea86 A. Beauville, Sur l'anneau de Chow d'une variété abélienne, Math. Ann. 273 (1986), 647-651.

Cer83 G. Ceresa, $C$ is not algebraically equivalent to $C^{-}$in its Jacobian, Ann. of Math. (2) 117 (1983), 285-291.

CG93 E. Colombo and B. van Geemen, Note on curves in a Jacobian, Compositio Math. 88 (1993), 333-353.

Col75 A. Collino, Poincaré's formulas and hyperelliptic curves, Atti Accad. Sci. Torino 109 (1975), 89-101.

Fak96 N. Fakhruddin, Algebraic cycles on generic abelian varieties, Compositio Math. 100 (1996), 101-119.

Mat61 A. Mattuck, Symmetric products and Jacobians, Amer. J. Math. 83 (1961), 189-206.

Nor89 M. Nori, Cycles on the generic abelian threefold, Proc. Indian Acad. Sci. Math. Sci. 99 (1989), 191-196.

Arnaud Beauville beauville@math.unice.fr Institut Universitaire de France et Laboratoire J.-A. Dieudonné (UMR 6621 du CNRS), Université de Nice, Parc Valrose, F-06108 Nice cedex 2, France 